PHYSICAL REVIEW D 94, 124010 (2016)

\title{
Comparison of methods for the detection of gravitational waves from unknown neutron stars
}

\author{
S. Walsh, ${ }^{1,2,3,{ }^{*}}$ M. Pitkin, ${ }^{4}$ M. Oliver, ${ }^{5}$ S. D'Antonio ${ }^{6}$ V. Dergachev,${ }^{7}$ A. Królak,${ }^{8}$ P. Astone, ${ }^{9}$ M. Bejger, ${ }^{10}$ \\ M. Di Giovanni, ${ }^{11,12}$ O. Dorosh ${ }^{13}$ S. Frasca, ${ }^{14}$ P. Leaci, ${ }^{14,9}$ S. Mastrogiovanni, ${ }^{14,9}$ A. Miller, ${ }^{14,15}$ \\ C. Palomba, ${ }^{9}$ M. A. Papa,${ }^{1,2,3}$ O. J. Piccinni, ${ }^{14,9}$ K. Riles, ${ }^{16}$ O. Sauter, ${ }^{16}$ and A. M. Sintes ${ }^{5}$ \\ ${ }^{1}$ Max Planck Institute for Gravitational Physics (Albert Einstein Institut), \\ Am Mühlenberg 1, 14476 Golm, Germany \\ ${ }^{2}$ Max Planck Institute for Gravitational Physics (Albert Einstein Institut), \\ Callinstrasse 38, 30167 Hannover, Germany \\ ${ }^{3}$ Department of Physics, University of Wisconsin, Milwaukee, Wisconsin 53201, USA \\ ${ }^{4}$ SUPA, School of Physics and Astronomy, University of Glasgow, Glasgow G12 8QQ, United Kingdom \\ ${ }^{5}$ Department de Física-IAC3, Universitat de les Illes Balears and Institut d'Estudis Espacials de \\ Catalunya, Cra. Valldemossa km. 7.5, E-07122 Palma de Mallorca, Spain \\ ${ }^{6}$ INFN, Sezione di Roma 2, Via della Ricerca Scientifica, 1, I-00133 Roma, Italy \\ ${ }^{7}$ LIGO Laboratory, California Institute of Technology, MS 100-36, Pasadena, California 91125, USA \\ ${ }^{8}$ Institute of Mathematics, Polish Academy of Sciences, Śniadeckich 8, 00-956 Warszawa, Poland \\ ${ }^{9}$ INFN, Sezione di Roma, P.le A. Moro, 2, I-00185 Roma, Italy \\ ${ }^{10}$ Nicolaus Copernicus Astronomical Center, Polish Academy of Sciences, \\ Bartycka 18, 00-716 Warszawa, Poland \\ ${ }^{11}$ INFN, Trento Institute for Fundamental Physics and Applications, I-38123 Povo, Trento, Italy \\ ${ }^{12}$ Università di Trento, Dipartimento di Fisica, I-38123 Povo, Trento, Italy \\ ${ }^{13}$ National Centre for Nuclear Research, 05-400 Otwock, Świerk, Poland \\ ${ }^{14}$ Università di Roma "La Sapienza,", I-00185 Roma, Italy \\ ${ }^{15}$ University of Florida, Gainesville, Florida 32611, USA \\ ${ }^{16}$ University of Michigan, 450 Church Street, Ann Arbor, Michigan 48109, USA
}

(Received 3 June 2016; published 8 December 2016)

\begin{abstract}
Rapidly rotating neutron stars are promising sources of continuous gravitational wave radiation for the LIGO and Virgo interferometers. The majority of neutron stars in our galaxy have not been identified with electromagnetic observations. All-sky searches for isolated neutron stars offer the potential to detect gravitational waves from these unidentified sources. The parameter space of these blind all-sky searches, which also cover a large range of frequencies and frequency derivatives, presents a significant computational challenge. Different methods have been designed to perform these searches within acceptable computational limits. Here we describe the first benchmark in a project to compare the search methods currently available for the detection of unknown isolated neutron stars. The five methods compared here are individually referred to as the PowerFlux, sky Hough, frequency Hough, Einstein @ Home, and time domain $\mathcal{F}$-statistic methods. We employ a mock data challenge to compare the ability of each search method to recover signals simulated assuming a standard signal model. We find similar performance among the four quick-look search methods, while the more computationally intensive search method, Einstein@Home, achieves up to a factor of two higher sensitivity. We find that the absence of a second derivative frequency in the search parameter space does not degrade search sensitivity for signals with physically plausible second derivative frequencies. We also report on the parameter estimation accuracy of each search method, and the stability of the sensitivity in frequency and frequency derivative and in the presence of detector noise.
\end{abstract}

DOI: 10.1103/PhysRevD.94.124010

\section{INTRODUCTION}

Continuous gravitational waves (CWs) from isolated neutron stars (NSs) are a potential source of detectable

*sinead.walsh@aei.mpg.de

Published by the American Physical Society under the terms of the Creative Commons Attribution 3.0 License. Further distribution of this work must maintain attribution to the author(s) and the published article's title, journal citation, and DOI. gravitational waves. $\mathrm{CW}$ radiation is emitted by rotating NSs with nonaxisymmetric deformations. The signal is expected to be relatively stable over many years. While the amplitude of CW signals is expected to be small, the continuous nature of the signal allows us to integrate the signal over large time spans of data to distinguish it from noise.

Broadband all-sky searches cover the whole sky over a broad range of frequencies and frequency derivatives in order to detect $\mathrm{CW}$ radiation from unknown NSs. 
All-sky searches in the initial Laser Interferometer Gravitational-Wave Observatory (LIGO) [1,2] and Virgo [3] data have so far not resulted in detection. Instead upper limits have been placed on the amplitude of CWs from isolated NSs [4-8]. The advanced detectors, which began operation in 2015, will eventually have a sensitivity to these weak signals over an order of magnitude better than that of the previous generation, with the largest gains at frequencies below $100 \mathrm{~Hz}$.

The purpose of the study presented in this article is to examine and compare the efficiency of the methods that will be used to perform all-sky searches in data from the advanced LIGO and Virgo detectors. In particular, we show the performance of the searches at this time. While it is appealing to think of software development as the implementation of a specific algorithm, in reality the algorithms we use are strongly influenced by the available data (and artifacts in it) as well as by computational hardware. The performance is also often limited by the available person power. Thus we do not compare optimal algorithm performance in some asymptotic limit (such as could be done for bubble sort vs quick sort), but rather we illustrate the performance that we can achieve right now, with the hope and expectation of further improvements. There have been similar efforts to compare search methods for CWs from a binary system where the sky position is known, but additional search parameters are required compared to searches for signals from isolated sources [9].

This comparison is made using a mock data challenge (MDC), for the standard CW signal model described in Secs. II and IV. In a blind all-sky search, detectable CW signals may deviate from this model, for example, if the NS glitches. For an accurate comparison of the all-sky search methods, further studies will be needed, which include deviations from the standard CW model. The study presented here serves as a first benchmark for the search methods, assuming the signal consistently follows the model.

A brief overview of the search methods is presented in Sec. III; the search parameters for the various searches are presented in Sec. VI. Section V describes how the methods are compared. The results of the comparison are presented in Section VII.

\section{THE SIGNAL}

Gravitational waves (GWs) emitted from nonaxisymmetric NSs are typically described by a signal model that remains relatively stable over years of observation [10]. The strain amplitude of the $\mathrm{GW}$ is proportional to the ellipticity, $\varepsilon$, defined as

$$
\varepsilon=\frac{\left|I_{x x}-I_{y y}\right|}{I_{z z}},
$$

where $I_{z z}$ is the principal moment of inertia of the star, and $I_{x x}$ and $I_{y y}$ are the moments of inertia about the axes. The strain amplitude of the $\mathrm{GW}$ at the detector, assuming a rigidly rotating triaxial body, is then given by

$$
h_{0}=\frac{4 \pi^{2} G}{c^{4}} \frac{I_{z z} f^{2} \varepsilon}{d}
$$

where $f$ is the frequency of the GW, $G$ is Newton's constant, $c$ is the speed of light, and $d$ is the distance to the NS. For a star steadily rotating around a principal axis of inertia, the frequency of the GW is at twice the rotational frequency of the NS. The frequency evolves over time as energy is lost due to various dissipation mechanisms, including $\mathrm{GW}$ emission. The first time derivative of the frequency, $\dot{f}$, is referred to as spin-down.

The signal model is described by eight parameters, four phase evolution parameters $\left(f_{0}, \dot{f}, \alpha, \delta\right)$ and four amplitude parameters $\left(h_{0}, l, \psi, \phi_{0}\right)$, where $\iota$ is the inclination angle between the line of sight to the NS and its rotation axis, $\psi$ is the polarization angle, and $\phi_{0}$ is the initial phase of the signal at a reference time.

The parameter $f_{0}$ is the GW frequency at a reference time $t_{0}$. The GW frequency in the Solar System barycenter (SSB) at time $t$ is given by

$$
f_{\mathrm{SSB}}(t)=f_{0}+\dot{f}\left(t-t_{0}\right),
$$

assuming the second time derivative of the frequency, $\ddot{f}$, is negligible. The frequency measured in the detector, $f(t)$, is shifted due to the Doppler effect from the motion of the Earth around the Sun and by the rotation of the Earth. The track of the signal in the time-frequency plane, as measured in the detector, is given by

$$
f(t)=f_{\mathrm{SSB}}(t)+f_{\mathrm{SSB}}(t) \frac{\mathbf{v}(t) \cdot \mathbf{n}}{c},
$$

where $\mathbf{v}(t)$ is the detector velocity with respect to the SSB frame and $\mathbf{n}$ is the unit vector pointing towards the sky location of the source.

In a blind all-sky search there is also the potential for the detection of signals produced by different source models (e.g., $r$-modes [11]). The ability of the all-sky search methods to recover such signals is not examined in this study. Here we assume the signal follows the model described above.

\section{CURRENT METHODS}

The most sensitive search for $\mathrm{CW}$ signals is performed with a fully coherent integration over a large time span of data. The computational power required for the integration increases rapidly with the observation time of the data. When searching for CW signals over a broad frequency and spin-down range, and over the whole sky, a fully coherent search quickly becomes computationally infeasible $[12,13]$.

This is the motivation for semicoherent search methods. The data are split into shorter segments that are analyzed 
coherently. Then, for each point in the parameter space $\left(f_{0}, \dot{f}, \alpha, \delta\right)$, the coherent results in each segment are combined incoherently to determine the signal-to-noise ratio (SNR) over the entire set of data. For limited available computing power, these semicoherent search methods achieve a higher sensitivity than could be achieved with a fully coherent search with a tractable coherence time [13].

Some searches use segments that are short enough, on the order of $1800 \mathrm{~s}$, so that the signal remains within a single Fourier frequency bin in each segment. In this case, the power of the GW signal is extracted with a single Fourier transform in each segment. This approach is computationally efficient, but the limit on the segment length limits the maximum sensitivity that can be reached by such a search, as sensitivity scales with coherent segment length. The "natural" frequency resolution of these searches is given by the inverse of the segment length. Other searches use longer segment times, hours to days, to increase the SNR of the signal in each segment. In this case, the coherent integration uses the more computationally demanding $\mathcal{F}$-statistic [10] to take into account signal modulation within the segment.

Each of the search methods uses a different variable to quantify SNR, so the numeric values of the SNR thresholds used by each search cannot be directly compared.

The sensitivity of the semicoherent searches is improved by taking a hierarchical approach. After the semicoherent all-sky search, candidates are selected with a threshold that is lower than needed to claim a detection. A refined, more sensitive search is then performed in the parameter space surrounding each candidate. In principle, the significance of recovered candidates increases in the presence of signal, but not if an original candidate is due to a random noise outlier. The increasingly refined hierarchical searches are continued until all candidates are excluded as noise or until the false alarm rate is reduced enough that any candidate that survives can be considered a detection.

The hierarchical refinement stages are designed such that any signal passing the first stage has a high probability of being recovered at each following stage. Therefore, the threshold at the first stage ultimately determines the sensitivity of the search.

The deepest searches are performed by the Einstein@Home pipeline, which benefits from the large computing power provided by the Einstein@ Home project (Sec. III D). Einstein@Home searches take many months before the presence of signal can be confirmed or excluded. There are also quick-look search pipelines that have a much shorter turnaround time. Each all-sky search makes different tradeoffs in sensitivity vs robustness against deviations from the assumed signal model. In the following we provide a brief overview of the search procedure employed by each pipeline, and the distinguishing characteristics of each method.

\section{A. PowerFlux}

The PowerFlux method is described in $[5,14,15]$. The search uses 1800-s Hann-windowed short Fourier transforms (SFTs), with an effective coherence length on the order of $900 \mathrm{~s}$. For the semicoherent search, the power from each SFT is recorded at the frequency determined by Eq. (4) for each point in parameter space, where $t$ is the time at the center of the SFT. In this search an offset in frequency is added to Eq. (4) to allow for sampling frequencies with a resolution below the natural resolution of a single SFT.

The power is then weighted, according to noise and the detector antenna pattern, to reduce outliers from noise artifacts and maximize the SNR. In addition to searching over the four-dimensional parameter space $(f, \dot{f}, \alpha, \delta)$, PowerFlux also searches over polarization angle $\psi$ by incorporating the amplitude expected for a particular polarization into the SFT power weighting.

The data set is partitioned into $\sim 1$ month stretches to look for signals that may be present in only part of the data. For each point in parameter space, the sum of the weighted power (in each SFT) is produced for every contiguous combination of 1 month stretches. High-SNR candidates are identified based on their persistence across contiguous stretches of data.

The candidates are then confirmed as signal or rejected as noise with four hierarchical search stages around each candidate. The parameter space refinement increases with each stage, and the last three stages use the loosely coherent detection pipeline [16].

\section{B. Sky Hough}

The sky Hough method is described in $[8,14]$. For this search, 1800-s SFTs are digitized by replacing their spectra with a collection of ones and zeros called a peak-gram. Each frequency bin gets a value of one if the normalized SFT power exceeds 1.6, and zero otherwise. The Hough number count is calculated in the semicoherent part of the search. This is the weighted sum of the ones and zeros of the different peak-grams along the track corresponding to each point in parameter space in the time-frequency plane, where the frequency in each segment is determined by Eq. (4). This sum is weighted based on the detector antenna pattern and the noise level, to suppress outliers from detector artifacts. Candidates are selected based on the deviation of the weighted number count from its value in Gaussian noise.

The data are split into two sets containing an equal number of SFTs. The search parameter space is split into subregions in frequency and sky location. For every region the search returns a list of the most significant candidates (a toplist) for both data sets. These candidates are required to pass a significance threshold, and a $\chi^{2}$ test is applied to eliminate candidates coming from detector artifacts. Candidates that are not within a coincidence window of each other in both data sets are discarded. 
A clustering algorithm is then applied to coincident candidates. The most significant cluster candidate per $0.1 \mathrm{~Hz}$ is chosen based on its distance to all other candidates in the toplist, weighted by some significance. Passing candidates are confirmed or rejected with a refinement stage, which covers a reduced parameter space around each candidate with higher resolution in spin-down and sky location.

\section{Time domain $\mathcal{F}$-statistic}

The time domain $\mathcal{F}$-statistic search method uses the algorithms and pipeline described in $[7,17]$. This analysis uses fast Fourier transformed data segments of 2 sidereal days each, split into bands of $0.25 \mathrm{~Hz}$. The coherent $\mathcal{F}$-statistic, which accounts for signal modulations due to spin-down and Doppler shift, is calculated in each segment for every point in parameter space. Candidates with an $\mathcal{F}$-statistic above threshold are recovered. Recovered candidates around known detector artifacts are vetoed, as are those with similar profiles to stationary noise lines and those close to the polar caps in equatorial coordinates.

For the semicoherent part of the search, the method searches for coincidences among candidates in each 2-day segment. Candidate frequencies are converted to a common reference time, $t_{0}$, using the candidate spin-down as in Eq. (3). Coincident candidates are counted, within a coincidence window large enough to account for Doppler modulation. The coincidence with the highest multiplicity is considered the most significant candidate, which is then selected or rejected based on a threshold on its false alarm probability.

\section{Einstein@Home}

Einstein@Home is a volunteer-driven distributed computing project where members of the public donate their idle computing power to the search for GWs [18]. The donated computing power allows for broader and more sensitive searches for CWs. The Einstein@Home search is described in [4]. The search begins with 1800-s SFTs. SFT frequency bins that overlap with known detector artifacts are cleaned by replacing them with Gaussian noise. For the coherent analysis, the SFTs are combined into segments of a few days. The $\mathcal{F}$-statistic is computed for each segment and for each parameter space point.

An average $2 \mathcal{F}$-statistic is then calculated by summing the $2 \mathcal{F}$ values in each segment. This summing is done on a grid in parameter space that is finer in spin-down than the parameter space used for the coherent analysis. In each segment, for each point on the finer grid, the closest point on the coarse grid is determined using the global correlation transform method [19]. The $2 \mathcal{F}$ at the closest coarse grid point in each segment is summed to approximate the average $2 \mathcal{F}$ at a given fine grid point across the entire data set. The logBSGL statistic, described in [20], is calculated for each fine-grid point. This is derived from the $2 \mathcal{F}$-statistic, and suppresses detector artifacts appearing in one detector.

The search parameter space is split into regions in frequency and sky. The search is performed for each region, and a toplist of the candidates ranked by $\operatorname{logBSGL}$ is returned. Candidates from the toplist are selected for further study based on their $2 \mathcal{F}$ value. The threshold applied depends on the total number of candidates above this threshold. There must be few enough that they can all be studied further with a refined search. These candidates are expected to be predominantly from Gaussian noise.

The search proceeds with multiple hierarchical stages of refinement, described in [21], to confirm or reject the presence of a signal.

\section{E. Frequency Hough}

The frequency Hough method is described in [6,22,23]. The analysis uses time-domain cleaned SFTs with a time span that depends on the frequency band of the search and is chosen so that the signal remains within a single Fourier frequency bin. A time-frequency map (peakmap) is constructed from the database by selecting the most significant local maxima on the square root of the equalized power, ${ }^{1}$ called peaks, over a threshold of $\sqrt{2.5} \simeq 1.58$.

The peakmap is cleaned by removing peaks corresponding to lines at a fixed frequency and to wandering lines. Because of time constraints, the cleaning of wandering lines is not applied in the MDC.

In the semicoherent frequency Hough step, for each signal frequency $f_{0}$, the time-frequency points of the corrected peakmap $f(t)$ are mapped onto the signal frequency-spin-down plane for every sky position, using Eq. (4) and taking into account the frequency bin width. The search parameter space (sky position, frequency, spindown) is suitably discretized. In particular, the frequency resolution is increased by a factor of ten with respect to the natural choice (given by the inverse of the SFT duration). For each sky position, this process results in a Hough number count on the signal frequency-spin-down plane. The adaptive procedure on the Hough transform, which would allow us to take into account noise nonstationarity, has not been used in this analysis.

A given number of the most significant candidates are selected at each sky position and in each $1-\mathrm{Hz}$ interval. This avoids being blinded by particularly disturbed frequency bands. For each candidate a search, refined in spin-down and sky resolution, is performed around the candidate parameters. The refined candidates are clustered and their coincidences with the candidates of another data set are

\footnotetext{
${ }^{1}$ The equalized power is obtained by dividing the periodogram squared by an autoregressive estimation of the average power. The result is a quantity that typically has a value close to unity except in correspondence of narrow spectral lines, where it takes values larger than one.
} 
computed. The coincident candidates are then ranked by significance and the most significant candidates are subject to a refinement stage.

\section{THE MOCK DATA CHALLENGE}

The aim of the MDC is to empirically compare the performance of current all-sky search methods when searching for a standard CW signal from an isolated NS. This is done by simulating the detector response to $\mathrm{CW}$ signals in data from the S6 LIGO science run [24], with software injections at a range of frequencies. Each of the pipelines described in Sec. III then performs a search over the data to assess their ability to recover this signal.

\section{A. The data}

The MDC search is performed over data from the LIGO S6 science run, in which simulated CW signals are injected. Real LIGO data are used to assess the performance of search methods in the presence of detector artifacts. The software injections are generated with lalapps sw inj_frames in the LALSuite software package [25]. There are 3110 injections in total. In general, the SNR of the injections was drawn randomly from a uniform distribution between 0 and 150 for a coherent single detector analysis in S6 H1 data (15 months). 50 of the injections have a coherent SNR between 1000 and 2000 .

Between 40 and $1550 \mathrm{~Hz}$, the injections are placed at $0.5 \mathrm{~Hz}$ intervals, while 90 further injections between 1550 and $2000 \mathrm{~Hz}$ are placed at $5 \mathrm{~Hz}$ intervals. The sky position is isotropically distributed over the celestial sphere. The spin-down is randomly drawn from a uniform distribution in $\log$ space between $-1 \times 10^{-9}$ and $-1 \times 10^{-18} \mathrm{~Hz} / \mathrm{s}$ for $95 \%$ of injections and between $1 \times 10^{-18}$ and $1 \times 10^{-13} \mathrm{~Hz} / \mathrm{s}$ for $5 \%$ of injections. ${ }^{2}$ A braking index between $n=5$ and $n=7$ [ $n$ defined implicitly in Eq. (5)] is applied to $25 \%$ of the pulsars with spin-down. The braking index of $n=5$ is for a NS that loses all its rotational energy through emission from a constant mass quadrupole, while $n=7$ is for saturated $r$-mode emission [11,26,27]. From the braking index, and the assigned frequency and frequency derivative, the second and third frequency derivative are assigned via the equation

$$
\ddot{f}=\frac{n \dot{f}^{2}}{f}, \quad \dddot{f}=\frac{n \dot{f}}{f}\left(2 \ddot{f}-\frac{\dot{f}^{2}}{f}\right) .
$$

The nuisance parameters of the NS, $\psi, \phi_{0}$, and $\cos l$, are randomly drawn from uniform distributions with the ranges $[-\pi / 4, \pi / 4],[0,2 \pi]$, and $[-1,1]$, respectively.

\footnotetext{
${ }^{2}$ Some injections have been given spin-ups as real signals can have observed (rather than necessarily intrinsic) spin-ups due to, e.g., large proper motions for nearby sources, or accelerations in the centers of globular clusters.
}

\section{B. The MDC search}

It is not feasible for all pipelines to perform a full all-sky search for the MDC, as limited computational resources must be reserved for searches for real signals. Instead, the search is performed over a reduced volume in parameter space around each injection. This MDC search volume is chosen to be small enough that even the most computationally expensive pipeline can participate in the MDC, and large enough so that the MDC results will accurately represent the result of a full all-sky search.

Each injection is placed roughly at the center of a MDC search volume of $0.1 \mathrm{~Hz}$, between $\min \left[-2 \times 10^{-9} \mathrm{~Hz} / \mathrm{s}\right.$, $-3 \times$ true spin-down/spin-up $]$ and $\max \left[2 \times 10^{-9} \mathrm{~Hz} / \mathrm{s}\right.$, $-3 \times$ true spin-down/spin-up] and a region in the sky with a radius of 30 degrees $\times \min [200 \mathrm{~Hz} /$ injection frequency, 1]. The pipelines are provided with the start point and width of the bands in frequency and frequency derivative, and the center and radius of the sky patch to be searched.

The specifics of the MDC search performed by each pipeline are described in Sec. VI. After the analysis, each pipeline provides a list of candidates considered to be detections, with the candidate frequency, spin-down, right ascension, declination, and the reference time at which the frequency is specified.

The MDC is split into two stages, with each stage covering half of the signals. In the first stage the signal parameters are known before performing the search. Some search methods use this stage to tune their search parameters, such as the search grid spacing and the candidate selection thresholds. It is normal to tune some search parameters before a search over real data, as the optimal search parameters will depend on the properties of the data. In the second stage of the MDC, the signal parameters are unknown; only the boundaries of the search region for each injection are provided. These are referred to as blind injections. This stage is used to verify that the results from the search over known injections are not biased. Once verified, the results from all injections are combined for the final comparison between pipelines.

\section{Defining detections}

The search parameters and selection criteria used in allsky searches depend on the parameter space to be covered. For the MDC, each search is required to use search parameters, significance thresholds, and selection criteria that would be used in a search over the complete parameter space in frequency, spin-down, and sky.

In an all-sky search, all candidates selected from the initial all-sky search would pass through the hierarchical refinement stages. The number of candidates that can be followed up is limited, and it is different for each method. The detection threshold applied in the MDC must be shown to result in a manageable number of candidates from noise in a full all-sky search for each pipeline. Therefore, each 
pipeline must establish the number of false alarms expected in a full all-sky search with the thresholds applied in the MDC. This false alarm rate indicates the number of candidates that will be passed to the hierarchical search stages. It is expected that no candidates from noise would survive all hierarchical search stages, with a false alarm rate which is low enough that surviving candidates can be considered detections.

As mentioned in Sec. III, the selection criteria applied after the initial search ultimately determine the ability to recover signals, as the refinement stages primarily exclude noise. To avoid excessive computing costs, we determine which candidates would result in a detection without requiring them to pass through all refinement stages. For the sky Hough and time domain $\mathcal{F}$-statistic searches, no false alarms from random noise outliers are expected among the candidates selected within the MDC search volume. Therefore, all candidates are considered detections. The PowerFlux candidates are passed through the first refinement stage, after which no false alarms from random noise outliers are expected in the MDC, so all candidates after the first refinement stage are considered detections.

The Einstein@Home search does expect false alarms from random noise outliers after the initial search in the MDC, as it is set up to refine many more candidates than the other searches. The frequency Hough search also expects false alarms because it searches the whole parameter space, not just the reduced MDC search volume. In a real search, all of these candidates would be passed through the refinement stages, and candidates from signals would result in detections. In the MDC, we apply a threshold on the distance in parameter space between the signal and the recovered candidates in order to identify the candidates from the initial search that would result in a detection after the refinement stages.

For all pipelines, the refined searches are performed for a few MDC candidates that are close to the selection threshold to demonstrate that signals within this distance threshold are retained after the refinement. We also look at the distance in each dimension of parameter space between the signal and the recovered candidates to check for outliers.

\section{COMPARISON OF METHODS}

\section{A. Detection efficiency}

Here we are primarily concerned with the ability of different searches to recover the $\mathrm{CW}$ signals. The detection efficiency is the fraction of signals that are considered detected, and it is the benchmark that we will use to compare pipeline performance. The detection efficiency is measured as a function of signal strength, $h_{0}$, expressed by the sensitivity depth $\sqrt{S_{h}} / h_{0}(1 / \sqrt{\mathrm{Hz}})$, where $S_{h}$ is the harmonic sum over both detectors of the approximate harmonic mean power spectral density of the data, at the frequency of the signal. The harmonic mean is computed, based on inverse variance weighting, by averaging together ten frequency bins to either side of the central frequency bin to compute a mean noise level, which is assumed to equal the variance. The sensitivity of a search is expressed as its sensitivity depth at a fixed detection efficiency, with a more sensitive search having a higher sensitivity depth.

Some injections overlap with known detector artifacts. We examine the detection efficiency separately for these signals to assess the performance of the methods in noisy data. We also separate the detection efficiency by frequency, as S6 data contain fewer instrumental artifacts at frequencies greater than $400 \mathrm{~Hz}$. The detection efficiency is also assessed for signals with positive frequency derivative, and those with nonzero second order spin-down.

\section{B. Parameter estimation}

In a broad parameter space search, the reduction in parameter uncertainty is achieved through the hierarchical refinement stages that follow the original search. In the MDC the refinement stages are not systematically carried through, as explained in Sec. IV C. We examine the parameter uncertainty of detection candidates at the final MDC stage and discuss how each method plans to reduce this uncertainty to the level required for a confident detection. In most cases, this automatically yields good parameters estimation (see, e.g., [28]).

\section{Computational cost}

Each of the methods has made different compromises on sensitivity to develop an all-sky search using available computational resources. The available computing resources are comparable for all except the Einstein@Home search. The quick-look searches are designed to require 1-10 MSU (million standard units) of computing power, where one SU is one core-hour on a standard core, and the standard core used here is an Intel Xeon E5-2670 CPU (Sandy Bridge).

The Einstein@Home search is designed to run for months using the full computing resources of the Einstein@Home distributed computing project. The computing power used by the search depends on the number of volunteered CPUs, and the type of CPUs, but for comparison it is $\gtrsim 100 \mathrm{MSU}$. Due to the overhead involved in preparing an Einstein@Home search for distribution to volunteer computers, it is inefficient to run a search for $\lesssim 1$ month. Therefore, given the computing resources available to these searches, it would not make sense to develop a search that requires more computing resources than our quick-look searches but less than the Einstein@Home computing power.

The sensitivity of semicoherent search methods is expected to scale weakly with computing cost. The precise scaling is difficult to predict, as explained in [29], but is weaker than sensitivity depth $\propto C^{1 / x}$, where $C$ is the computing cost and $x$ is 5 or more. While this 
scaling is weak, the additional sensitivity provided by the Einstein@Home computing resources may make the difference between having no detection or detecting a $\mathrm{CW}$ from an isolated NS, which would have great scientific importance.

The best sensitivity for standard CW signals is ultimately limited by the available computing power. The high sensitivity of such a computationally intensive search comes at a cost: the sensitivity is easily lost due to deviations from the signal model, and when the data are disturbed by noise lines. In this situation, our heterogeneous approach to the search, with a number of differently optimal tools, has more potential than focusing on a single search method.

\section{IMPLEMENTATION}

In this section we detail the specific search parameters used by each pipeline for the MDC and explain why the thresholds chosen here are representative of the values in an all-sky search.

The search parameters and thresholds applied here will vary in future searches, depending on the observation length of available data, how well behaved the data are, and other factors. Any changes to these parameters will affect the detection efficiency. The effect of unforeseen improvements to the search methods are difficult to predict, and the variations will be different for each search method. When presenting the MDC results we consider only statistical uncertainties on the measured efficiency. One should keep in mind that these results are specific to the search implementation presented here.

Instances where the searches were not optimal in the MDC are highlighted. In some cases, predictions for how the sensitivity will change in future searches are included.

\section{A. PowerFlux}

The PowerFlux MDC search uses the same search parameters as the PowerFlux all-sky search over S6 data described in [15]; these are summarized in Table I. The S6

TABLE I. PowerFlux MDC search parameters. $\delta f$ and $\delta \dot{f}$ define the spacing between points on the frequency and spindown plane of the search grid respectively. The sky resolution is given by the sky refinement as shown in Eq. (6). The phase coherence, $\delta$, determines the amount of phase drift the methods allow between SFTs, with $\delta=0$ corresponding to a fully coherent case and $\delta=2 \pi$ corresponding to incoherent power sums.

\begin{tabular}{lcc}
\hline \hline Stage & 0 & 1 \\
\hline$T_{\text {coh }}(\mathrm{s})$ & 900 & 900 \\
$\delta f(\mathrm{~Hz})$ & $2.78 \times 10^{-4}$ & $6.95 \times 10^{-6}$ \\
$\delta f(\mathrm{~Hz} / \mathrm{s})$ & $2 \times 10^{-10}$ & $1 \times 10^{-10}$ \\
Sky refinement & 1 & 0.25 \\
Phase coherence $(\mathrm{rad})$ & $\mathrm{NA}$ & $\pi / 2$ \\
\hline \hline
\end{tabular}

search was not tuned for frequencies below $400 \mathrm{~Hz}$; therefore, a loss in performance at low frequencies is expected in the MDC. The tuning for searches in advanced detector data will include the low frequency range, reducing or removing this loss in performance.

The search uses all of the MDC data. The sky grid is isotropic on the celestial sphere, with the angular spacing between grid points given by the formula

$$
\frac{4500}{T_{\text {coh }} \times\left(f_{0}+f_{1}\right) \times 0.5 \times \text { sky refinement }},
$$

where $f_{0}$ and $f_{1}$ are the start and end frequencies of each $0.25 \mathrm{~Hz}$ band, and $T_{\text {coh }}$ is the coherent segment length. The sky refinement is a constant that is used to scale the resolution of the sky grid.

Every candidate with a SNR greater than 5 is selected. In order to pass to the first refinement stage, candidates are required to appear in at least six contiguous stretches of data. The number of false alarms in a real search is expected to be dominated by instrumental artifacts, and so it is difficult to predict. However, since the MDC search uses the same parameters as a previous all-sky search over S6 data, we know the number of false alarms is manageable [15].

Candidates surviving the first refinement stage would normally be passed through further stages, with less than $1 \%$ false dismissal in the subsequent stages. For the parameter space covered by the MDC, the false alarm rate after the first refinement stage is expected to be negligible. For the MDC, a signal is considered detected if a candidate survives stage one, as all MDC candidates at this stage are expected to be from signals. This choice is justified by performing all refinement stages for the weakest candidates and demonstrating that they are recovered with high significance close to the signal.

\section{B. Sky Hough}

The sky Hough MDC search uses a similar search grid to a previous all-sky search over LIGO data [8], given in Table II. The search uses all of the MDC data. The equatorial spacing of the sky grid points (in radians) is given by the formula

$$
\frac{10^{4} \delta f}{f \times \text { pixel factor }},
$$

TABLE II. Sky Hough MDC search parameters. The sky resolution is determined by the pixel factor as shown in Eq. (7).

\begin{tabular}{lc}
\hline \hline Tcoh $(\mathrm{s})$ & 1800 \\
$\delta f(\mathrm{~Hz})$ & $5.55 \times 10^{-4}$ \\
$\delta f(\mathrm{~Hz} / \mathrm{s})$ & $1.37 \times 10^{-11}$ \\
Pixel factor & 2 \\
\hline
\end{tabular}


where the pixel factor is a constant that is used to scale the resolution of the sky grid.

The postprocessing procedure has been updated significantly since the previous search, as described in [30] and briefly in Sec. III B. The most significant cluster candidate is required to have $\mathrm{SNR} \geq 4.5$, and pass the $\chi^{2}$ veto described in [8]. All surviving MDC candidates are considered detections.

By selecting only the most significant cluster per $0.1 \mathrm{~Hz}$ frequency band, there is an upper bound on the number of candidates from the all-sky search to follow up. Therefore, the number of surviving candidates would not be unreasonable in an all-sky search.

\section{Time domain $\mathcal{F}$-statistic}

The time domain $\mathcal{F}$-statistic search divides all of the MDC data into 2-day segments and uses 117 segments for the analysis based on the goodness of data [7]. The search configuration is summarised in Table III. The construction of the four-dimensional search grid is described in [17], to achieve a minimal match of $\sqrt{3} / 2$ using the smallest number of grid points with a frequency spacing of $5.79 \times 10^{-6} \mathrm{~Hz}$. The minimal match is the ratio of the optimal SNR, at the signal position, to the SNR at the search grid point closest to the signal in the worst case, i.e., when the signal is as far as possible from the nearest grid point in the four-dimensional search grid.

Candidates with $\mathcal{F}>10.5$ (corresponding to a SNR of 4.1) in each segment are selected. The bandwidth of each segment is $0.25 \mathrm{~Hz}$, but only candidates in the $0.1 \mathrm{~Hz}$ band defined by the MDC search are selected.

After counting coincidences across 2-day segments, the most significant candidate per $0.1 \mathrm{~Hz}$ band is considered a detection if it is coincident in at least 60 segments. With a simplified estimation of the false alarm rate, assuming Gaussian noise, this corresponds with a false alarm probability of less than $0.1 \%$ per $1 \mathrm{~Hz}$ band.

With only one candidate per $0.25 \mathrm{~Hz}$ band and an additional threshold with $0.1 \%$ false alarm probability, the number of false alarms will not become unmanageable in an all-sky search, and no false alarms are expected in the reduced parameter space covered by the MDC.

\section{Einstein@Home}

To cover the available frequency band of 40 to $2000 \mathrm{~Hz}$, the Einstein@Home search uses three separate search configurations for 40 to $500 \mathrm{~Hz}, 500$ to $1000 \mathrm{~Hz}$, and

TABLE III. Time domain $\mathcal{F}$-statistic search parameters. The minimal match is used to construct the four-dimensional search grid as described in [17].

\begin{tabular}{lc}
\hline \hline Tcoh $(\mathrm{h})$ & 48 \\
$\delta f(\mathrm{~Hz})$ & $5.79 \times 10^{-6}$ \\
Minimal match & $\sqrt{3} / 2$ \\
\hline \hline
\end{tabular}

TABLE IV. Einstein@Home MDC search parameters. The sky grid resolution is determined by the sky factor as shown in Eq. (8). The spin-down resolution used on the fine grid, for the semicoherent part of the search, is given by $\delta \dot{f}$ divided by the $\dot{f}$-refine value.

\begin{tabular}{lccc}
\hline \hline$f$ band $(\mathrm{Hz})$ & 40 to 500 & 500 to 1000 & 1000 to 2000 \\
\hline Tcoh $(\mathrm{h})$ & 60 & 60 & 25 \\
$\delta f(\mathrm{~Hz})$ & $3.61 \times 10^{-6}$ & $3.95 \times 10^{-6}$ & $7.75 \times 10^{-6}$ \\
$\dot{\delta f}(\mathrm{~Hz} / \mathrm{s})$ & $1.16 \times 10^{-10}$ & $1.83 \times 10^{-10}$ & $7.46 \times 10^{-10}$ \\
Sky factor & 0.01 & 0.04 & 0.07 \\
$\dot{f}$ refine & 230 & 230 & 150 \\
Min $2 \mathcal{F}$ & 6.17 & 6.17 & 5.56 \\
\hline \hline
\end{tabular}

1000 to $2000 \mathrm{~Hz}$. The search configurations are given in Table IV.

The searches use 9 months of MDC data. The sky grid is hexagonal and uniform on the ecliptic plane, with the distance between grid points given by

$$
\frac{\sqrt{\text { sky factor }}}{\pi \tau_{E} f},
$$

where $\tau_{E}$ is the radius of the Earth divided by the speed of light. The sky factor is a constant used to scale the resolution of the sky grid and is chosen empirically as described in [21]. The sky grid points are then projected to equatorial coordinates for the search.

The $2 \mathcal{F}$ thresholds given in Table IV are chosen to result in 35 million false alarms in Gaussian noise in an all-sky search for each frequency band. The false alarm rate in Gaussian noise is estimated as described in [4].

In an all-sky search, selected candidates are passed through the refinement stages. In the MDC, the parameter space around the candidates in the first refinement stage is used to determine which candidates from the initial stage would result in a detection; see Sec. IV C.

For the 40 to $500 \mathrm{~Hz}$ search, the first refinement stage searches $\delta f \pm 1.9 \times 10^{-4} \mathrm{~Hz}, \delta \dot{f} \pm 3.46 \times 10^{-11} \mathrm{~Hz} / \mathrm{s}$ and a sky patch with a radius of 1.2 initial-search sky grid bins around the selected candidate. Therefore, candidates within this region of the signal are considered detections. The 500 to $1000 \mathrm{~Hz}$ search uses a larger parameter space for the first refinement stage; candidates within $\delta f<6 \times 10^{-4} \mathrm{~Hz}$, $\delta \dot{f}<1.8 \times 10^{-10} \mathrm{~Hz} / \mathrm{s}$ and 3.2 sky grid bins of the signal are considered detections. The distance threshold is the same for the 1000 to $2000 \mathrm{~Hz}$ search, except that the candidate must be within 2.4 sky bins. In each case, 90\% of signals are expected to have a candidate within this region around the signal parameters.

\section{E. Frequency Hough}

The frequency Hough MDC search parameters are given in Table V. The sky grid is constructed in ecliptic 
TABLE V. Frequency Hough MDC search parameters.

\begin{tabular}{lc}
\hline \hline Tcoh $(\mathrm{s})$ & 1024 \\
$\delta f(\mathrm{~Hz})$ & $9.76 \times 10^{-4}$ \\
$\dot{\delta f}(\mathrm{~Hz} / \mathrm{s})$ & $2.4 \times 10^{-11}$ \\
\hline \hline
\end{tabular}

coordinates as described in [23] and is uniform in ecliptic longitude at fixed ecliptic latitude. In a real search the coherent segment length would typically be between 1024 and $8192 \mathrm{~s}$, depending on the frequency band. For the $\mathrm{MDC}$, it is restricted to $1024 \mathrm{~s}$ over the whole frequency band of the analysis to reduce the computational cost. This implies a sensitivity loss of up to a factor of $\sqrt{8}$ with respect to a real search in the lower frequency bands, where the coherent segment length would typically be higher.

The frequency Hough search is performed using all of the MDC data. For the MDC, the search is performed over the whole parameter space. This is because the analysis procedure depends on ranking the most significant candidates per frequency band, as opposed to applying a threshold, so replicating the detection rate of an all-sky search using a smaller parameter space is not trivial. The results for injections where the signal parameters are known are used to optimize the selection of candidates. The search over injections where the signal parameters are unknown is used to validate these results.

The four most significant candidates per $0.1 \mathrm{~Hz}$ frequency band are selected. The refinement stages are not performed for the MDC. Instead, a signal is considered detected if a candidate is recovered close enough that it is expected to survive the refinement stages. The distance is given by $d=\sqrt{\sum \delta_{n}^{2}}$, where $\delta_{n}$ is the difference between the signal and candidate parameter, expressed in units of search grid bins, for each dimension of parameter space $n=\left(f_{0}, \dot{f}, \alpha, \delta\right)$. A more complete description of this distance is given in [6]. Signals that have a candidate within a distance of 3 are considered detected.

\section{RESULTS}

\section{A. Detection efficiency}

The detection efficiency, measured on the combined results from the set of known injection and blind injections, is shown in Fig. 1. Figure 2 shows the detection efficiency separately when measured with injections where the injection parameters are known, and blind injections where the parameters are unknown. The two measurements agree; therefore, we combine the results from both injection sets. This reduces the statistical uncertainty on the measured detection efficiency.

The dependence of the detection efficiency on sensitivity depth is obtained with a sigmoidal fit to the MDC results. The uncertainty band around the resulting sigmoid is obtained by fitting sigmoids to the minimum and maximum of the binomial uncertainties (at the $1 \sigma$ level) on the

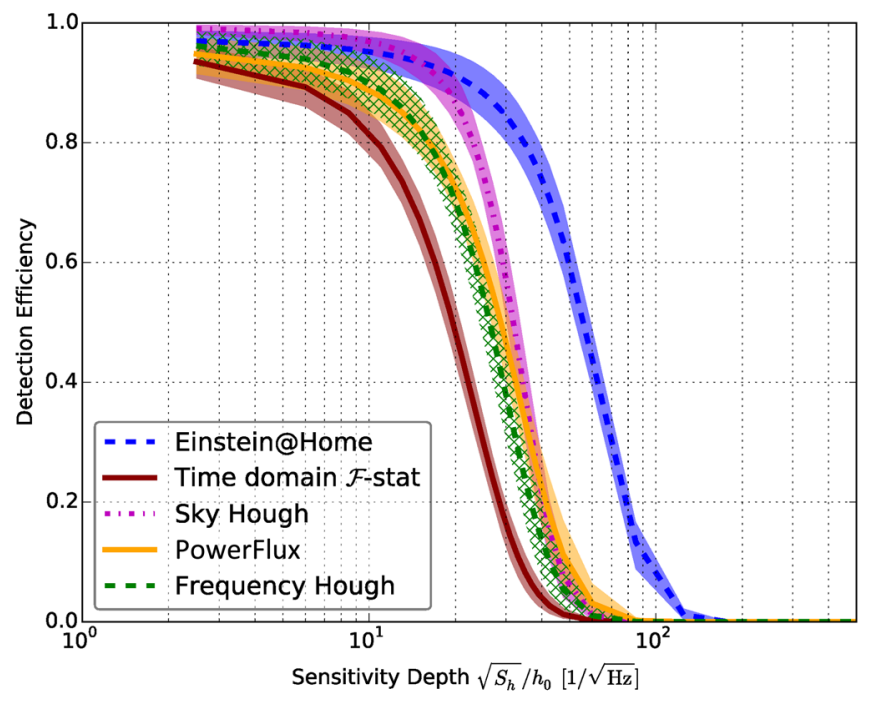

FIG. 1. Detection efficiency measured for all 3110 injections. The frequency Hough results are shown with a hatched error band because the efficiency is measured for a subset (1920) of the MDC injections. The curves and error bands are obtained by fitting sigmoids to the data; see Sec. VII A. The error bands represent the statistical uncertainty on the detection efficiency measured for the search implementation and data used in the MDC.

detection efficiency. The uncertainty band represents the statistical uncertainty on the detection efficiency for this particular search implementation over LIGO S6 data. We would expect to see variations in the measured efficiency with changes in the observation time of the data, quieter or noisier data, search improvements, and other changes expected in advanced detector data. When considering the results one should also keep in mind that these are for standard CW signals.

The frequency Hough results are complete up to $1000 \mathrm{~Hz}$; however, there are no results above this frequency because of technical difficulties with the computer cluster used to perform the search. Specifically, the duration of searches in some frequency bands exceeded the allowance of the cluster for some CPUs. This occurs more at higher frequencies due to the increase in sky grid templates. This issue is being resolved for the frequency Hough search, but not within the time scale of this MDC. The frequency Hough results are scaled so that the detection efficiency is measured for the subset of injections for which the search is complete. The results are displayed with a hatched uncertainty band to highlight the difference with respect to the other searches.

Figure 1 shows that the results from the frequency Hough, sky Hough, and PowerFlux searches are comparable. If we compare the sensitivity depth achieved at $60 \%$ efficiency we see that the time domain $\mathcal{F}$-statistic is less sensitive to these standard $\mathrm{CW}$ signals. At the same efficiency, the Einstein@ Home search is a factor of two more sensitive than the next most sensitive search. This difference can be 

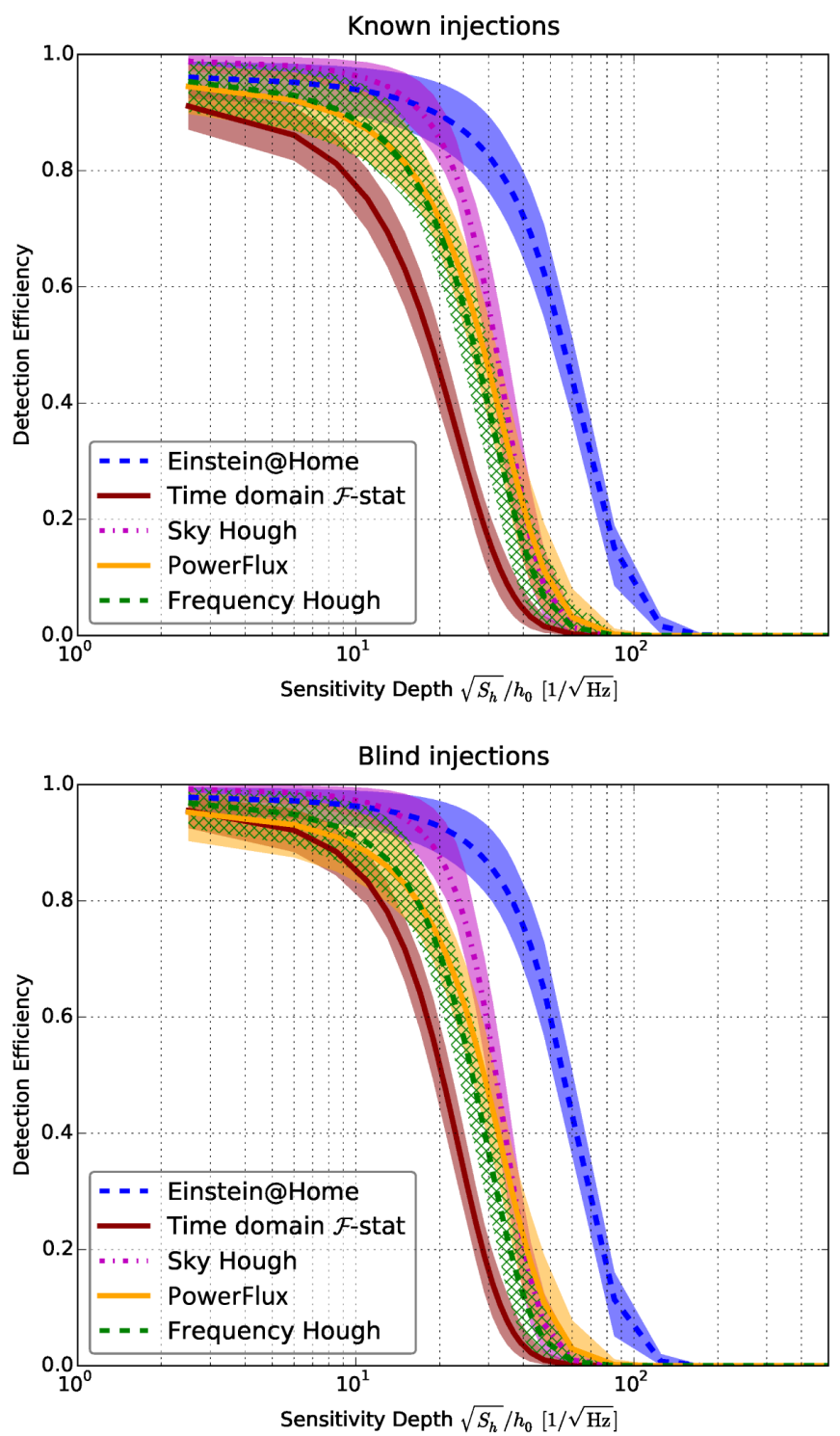

FIG. 2. Detection efficiency measured with 1561 known injections (top) and 1549 blind injections (bottom). The frequency Hough results are shown with a hatched error band because the efficiency is measured for a subset of the MDC injections (949 known injections, 971 blind injections). The curves and error bands are obtained by fitting sigmoids to the data; see Sec. VII A. The error bands represent the statistical uncertainty on the detection efficiency measured for the search implementation and data used in the MDC.

attributed to a combination of the significant computing resources of the Einstein@Home project, the longer coherent segment length, recent method improvements, and the intensive refinement procedure that allows for the follow-up of many candidates from the all-sky search.

In Fig. 1 it is clear that the detection efficiency does not reach $100 \%$ for very strong signals. For Einstein@Home, this is due to signals that overlap with known noise lines, as shown in Sec. VII A 1. For PowerFlux, this is due to signals below $400 \mathrm{~Hz}$, as shown in Sec. VII A 2 .

\section{Robustness in the presence of detector artifacts}

Each method has a different procedure for excluding candidates caused by detector artifacts, also known as noise lines, described in Sec. III. In Fig. 3 we separate the detection efficiency measured in quiet data, and the detection efficiency measured for injections whose frequency overlaps with known noise lines.

The top panel of Fig. 3 shows that the efficiency for the sky Hough, time domain $\mathcal{F}$-statistic, and frequency Hough
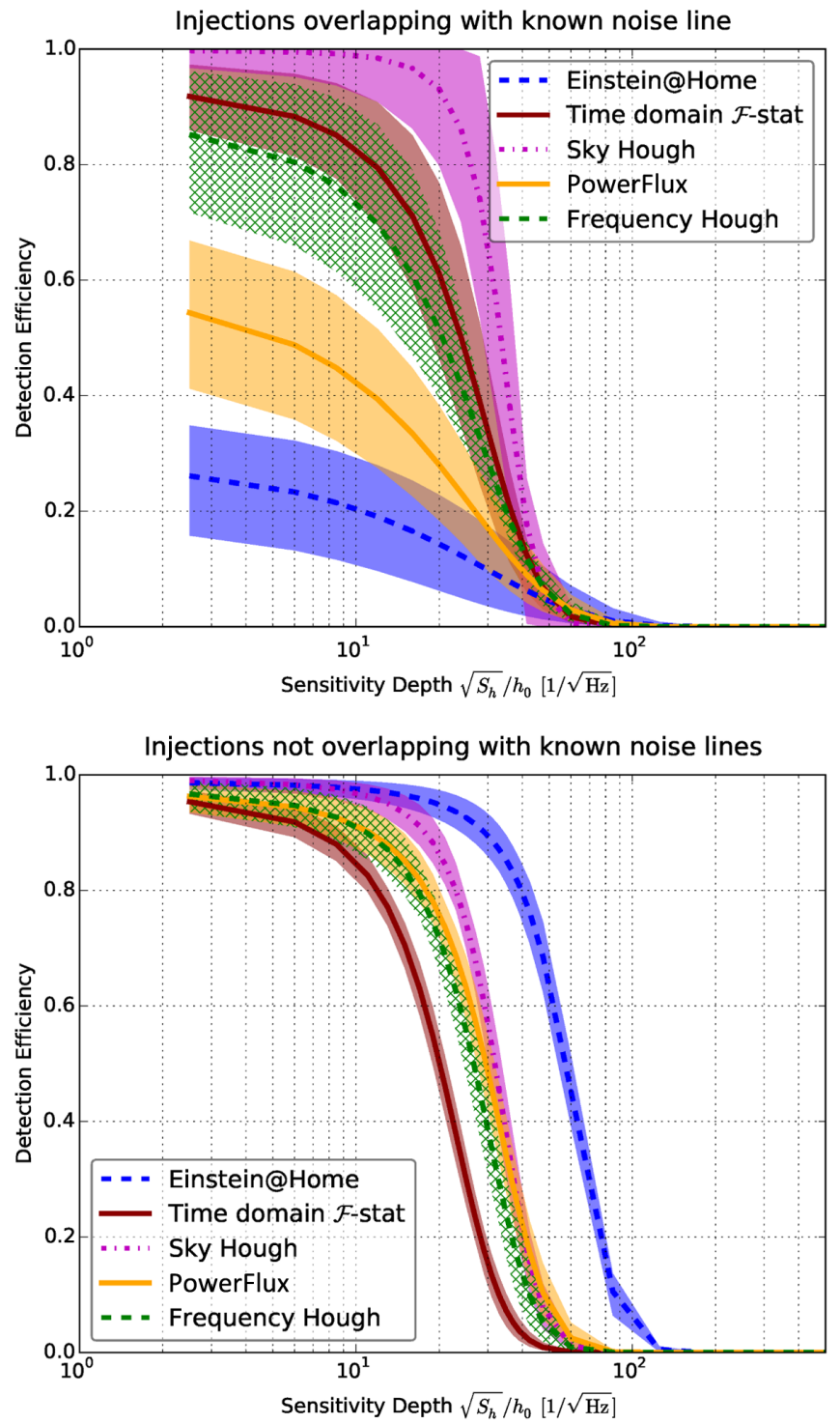

FIG. 3. Detection efficiency measured for injections overlapping with known noise lines (top, 184 injections), and when injections that overlap with known noise lines are excluded (bottom, 2926 injections). The frequency Hough results are shown with a hatched error band because the efficiency is measured for a subset of the MDC injections (top: 117, bottom: 1803). The curves and error bands are obtained by fitting sigmoids to the data; see Sec. VII A. The error bands represent the statistical uncertainty on the detection efficiency measured for the search implementation and data used in the MDC. 
searches remains unchanged, within the measurement uncertainty, in the presence of noise. The frequency Hough procedure for handling wandering lines is not included in the MDC; therefore, these results are not fully representative of noise handling in a real search.

As Einstein@Home applies an aggressive cleaning procedure, where known noise lines are replaced by Gaussian noise, any signal that overlaps with a noise line in both detectors is removed. When signal overlaps with a noise line in one detector, the signal is suppressed by the $\operatorname{logBSGL}$ statistic that downweights signals appearing in one detector. In the case of PowerFlux, signals overlapping with noise lines are suppressed by the procedure in which SFTs are weighted according to their noise level.

The bottom panel of Fig. 3 shows that, in the absence of known lines, the efficiency for strong signals has increased for PowerFlux and reaches almost 100\% for Einstein@Home.

\section{Dependence on signal frequency or spin-down}

Here we consider the detection efficiency only for injections that do not overlap with known noise lines. Figure 4 shows the detection efficiency separately for injections in the frequency ranges of 40 to $500 \mathrm{~Hz}, 500$ to $1000 \mathrm{~Hz}$, and 1000 to $1500 \mathrm{~Hz}$.

The sky Hough, time domain $\mathcal{F}$-statistic, and frequency Hough results do not depend on frequency. This indicates that the frequency Hough results would not change if injections above $1000 \mathrm{~Hz}$ were included. PowerFlux measures lower efficiency in the low frequency range. This is expected as the S6 analysis applied in the MDC was only tuned for signals above $400 \mathrm{~Hz}$. In the higher frequency bands, for which the search is designed, the detection efficiency approaches $100 \%$ for the strongest signals.

The drop in efficiency for the Einstein@Home search at higher frequencies is expected due to the choice of having equal computing cost assigned to the searches in each of the three frequency bands in Table IV. As the frequency increases, a higher sky-grid density, and therefore a higher computing cost, is required to achieve the same sensitivity. In order to keep the computing cost fixed, a coarser search grid is used in the higher frequency bands.

Figure 5 shows the detection efficiency for injections with large spin-down, small spin-down, and spin-up. There is no dependence on the frequency derivative of the signal for any of the searches.

\section{Dependence on signal second order spin-down}

A loss in detection efficiency is expected when the signal $\ddot{f}$ is greater than the $\ddot{f}_{\text {critical }}$ for a search, where $\ddot{f}_{\text {critical }}$ is given by

$$
\ddot{f}_{\text {critical }}=\frac{\delta f}{\mathrm{~T}_{\text {obs }}{ }^{2}}
$$
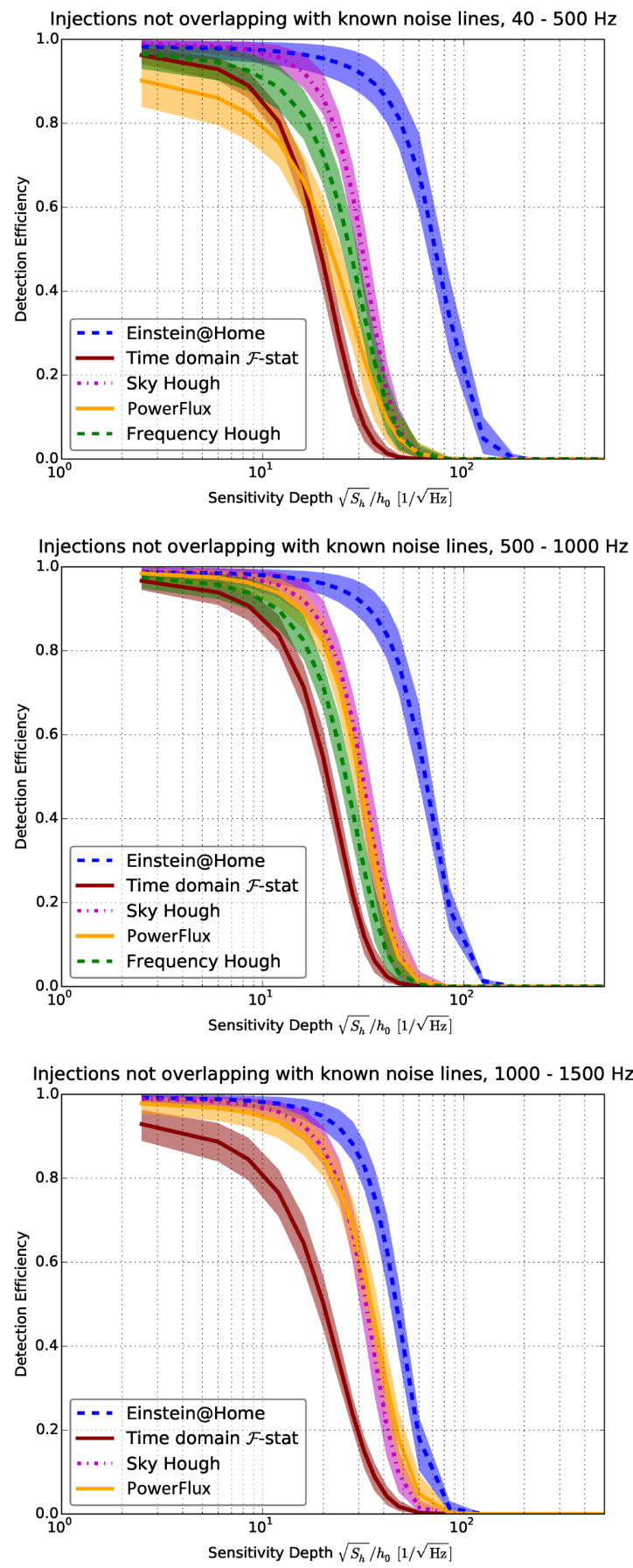

FIG. 4. Detection efficiency measured for injections in the frequency ranges of 40 to $500 \mathrm{~Hz}, 500$ to $1000 \mathrm{~Hz}$, and 1000 to $1500 \mathrm{~Hz}$ (859, 944, and 1123 injections, respectively). The frequency Hough results are complete for injections below $1000 \mathrm{~Hz}$. The curves and error bands are obtained by fitting sigmoids to the data; see Sec. VII A. The error bands represent the statistical uncertainty on the detection efficiency measured for the search implementation and data used in the MDC.

$\ddot{f}_{\text {critical }}$ is the value of $\ddot{f}$ at which the signal frequency will vary by more than a frequency bin, $\delta f$, over the observation time of the data, $\mathrm{T}_{\mathrm{obs}}$. In practice, the efficiency loss for $\ddot{f} \geq \ddot{f}_{\text {critical }}$ is expected to be mitigated to some degree by 


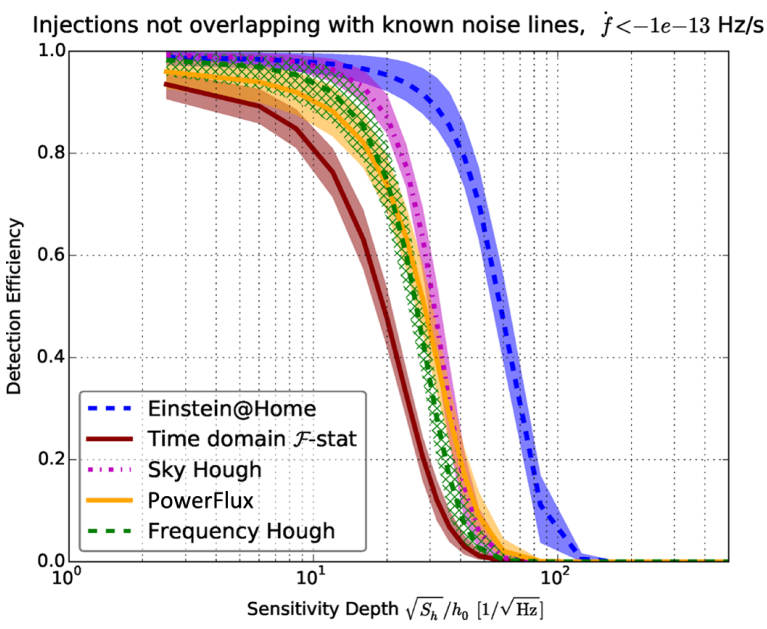

Injections not overlapping with known noise lines, $-1 e-13<f<=0 \mathrm{~Hz}$
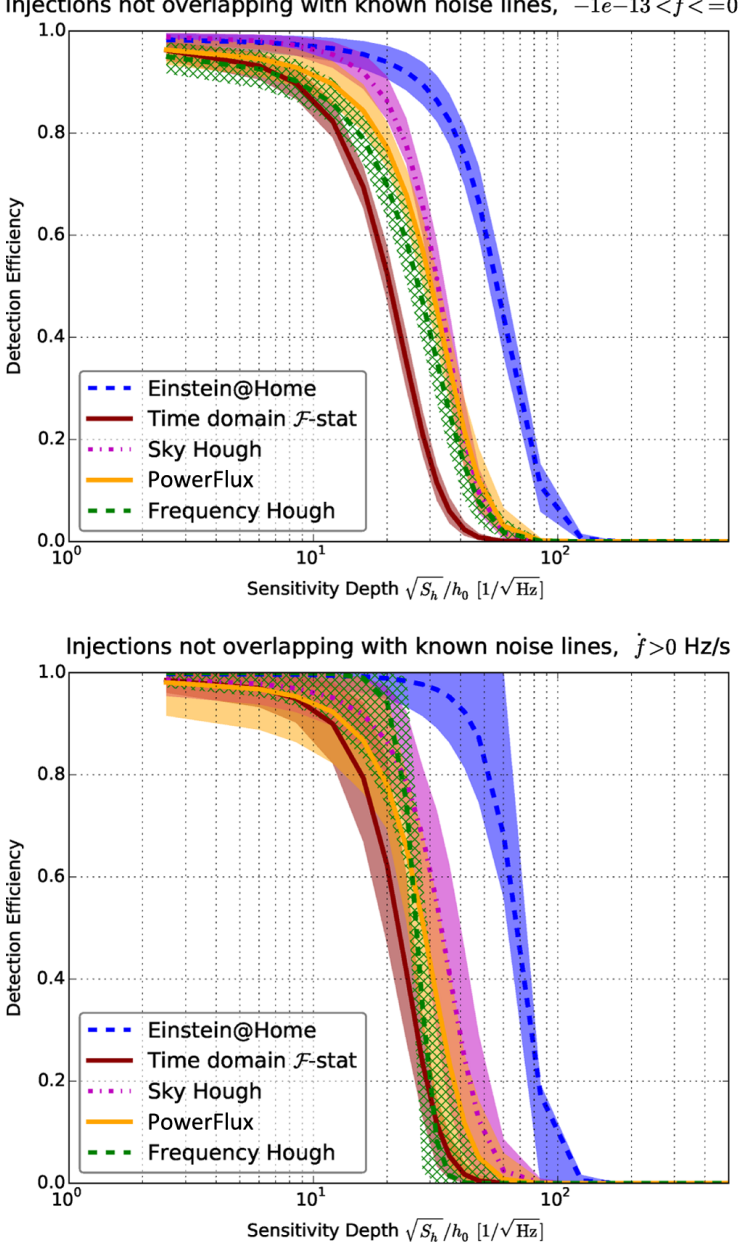

FIG. 5. Detection efficiency measured for injections with small spin-down $\left(<-1 \times 10^{-13} \mathrm{~Hz} / \mathrm{s}, 1260\right.$ injections $)$, large spindown $\left(-1 \times 10^{-13}\right.$ to $0 \mathrm{~Hz} / \mathrm{s}, 1517$ injections), and spin-up $(>0 \mathrm{~Hz} / \mathrm{s}, 149$ injections). Frequency Hough results are shown with a hatched error band because the efficiency is measured for a subset of the MDC injections (793, 919, and 457 injections, respectively). The curves and error bands are obtained by fitting sigmoids to the data; see Sec. VII A. The error bands represent the statistical uncertainty on the detection efficiency measured for the search implementation and data used in the MDC. apparent displacement of the signal parameters in the space of $\left(f_{0}, \dot{f}, \alpha, \delta\right)$.

Figure 6 shows the nonzero second order spin-down values of the MDC signals, for the range specified in Sec. IVA. The vertical lines show the $\ddot{f}_{\text {critical }}$ for each of the searches. Some signals have $\ddot{f} \geq \ddot{f}_{\text {critical }}$ for the Einstein@Home, time domain $\mathcal{F}$-statistic, and PowerFlux searches. However, in each case, there are too few signals with $\ddot{f} \geq \ddot{f}_{\text {critical }}$ to determine if they have an appreciable effect on the detection efficiency.

The stability of the detection efficiency for signals with $\ddot{f}>0$ is important as none of the pipelines searches explicitly over second order spin-down, and to do so would add a significant computational burden to the searches. Figure 7 shows that the detection efficiency is the same for signals with $\ddot{f}=0$ and $\ddot{f}>0$, with at least $99 \%$ of injections having $\ddot{f}<\ddot{f}_{\text {critical }}$. Due to the lack of injections with $\ddot{f} \geq \ddot{f}_{\text {critical }}$, the impact on detection from these injections was not examined.

For the sky Hough and frequency Hough searches, the impact of $\ddot{f} \geq \ddot{f}_{\text {critical }}$ is less of a concern for future searches, due to the short coherent segment length used by these searches. The $\ddot{f}_{\text {critical }}$ of the time domain $\mathcal{F}$-statistic search will be larger than the value in the MDC for the first advanced LIGO searches, which will have a lower $\mathrm{T}_{\mathrm{obs}}$ than the 15 months of the MDC data.

The PowerFlux $\ddot{f}_{\text {critical }}$ is calculated for refinement stage 1 in Table I. The $\delta f$ decreases in the next refinement stages, so $\ddot{f}_{\text {critical }}$ will decrease. The Einstein@Home search refinement stages also have reduced $\delta f$ and $\ddot{f}_{\text {critical }}$. Therefore, the impact on detection efficiency of $\ddot{f} \geq \ddot{f}_{\text {critical }}$ may warrant further study for these two searches.

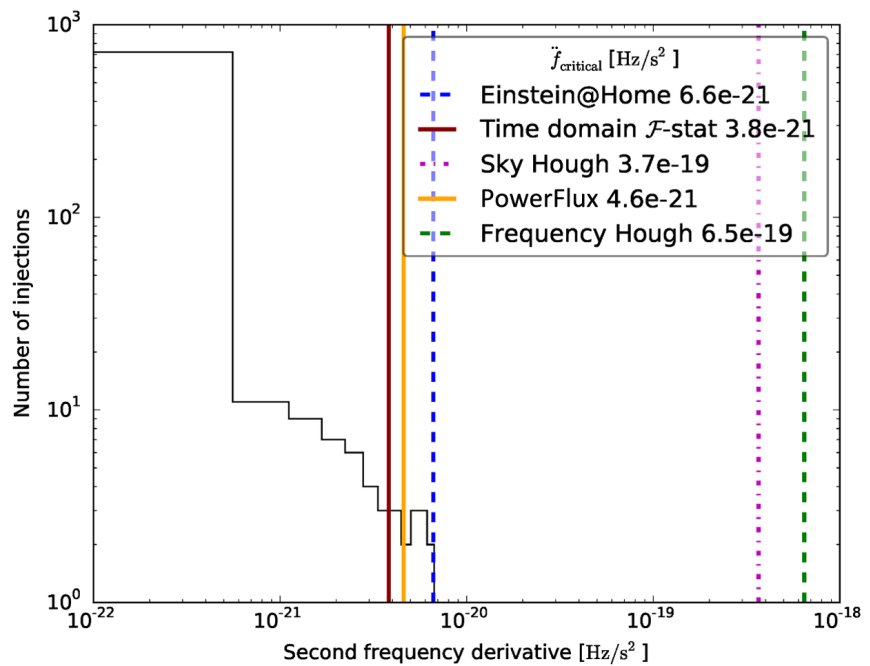

FIG. 6. Distribution of $\ddot{f}$ values for 781 injections with $\ddot{f}>0$. The vertical lines show $\ddot{f}_{\text {critical }}$ for each of the pipelines. The $\ddot{f}_{\text {critical }}$ is calculated after the first refinement stage for PowerFlux and for the 40 to $500 \mathrm{~Hz}$ search setup for Einstein@Home. 

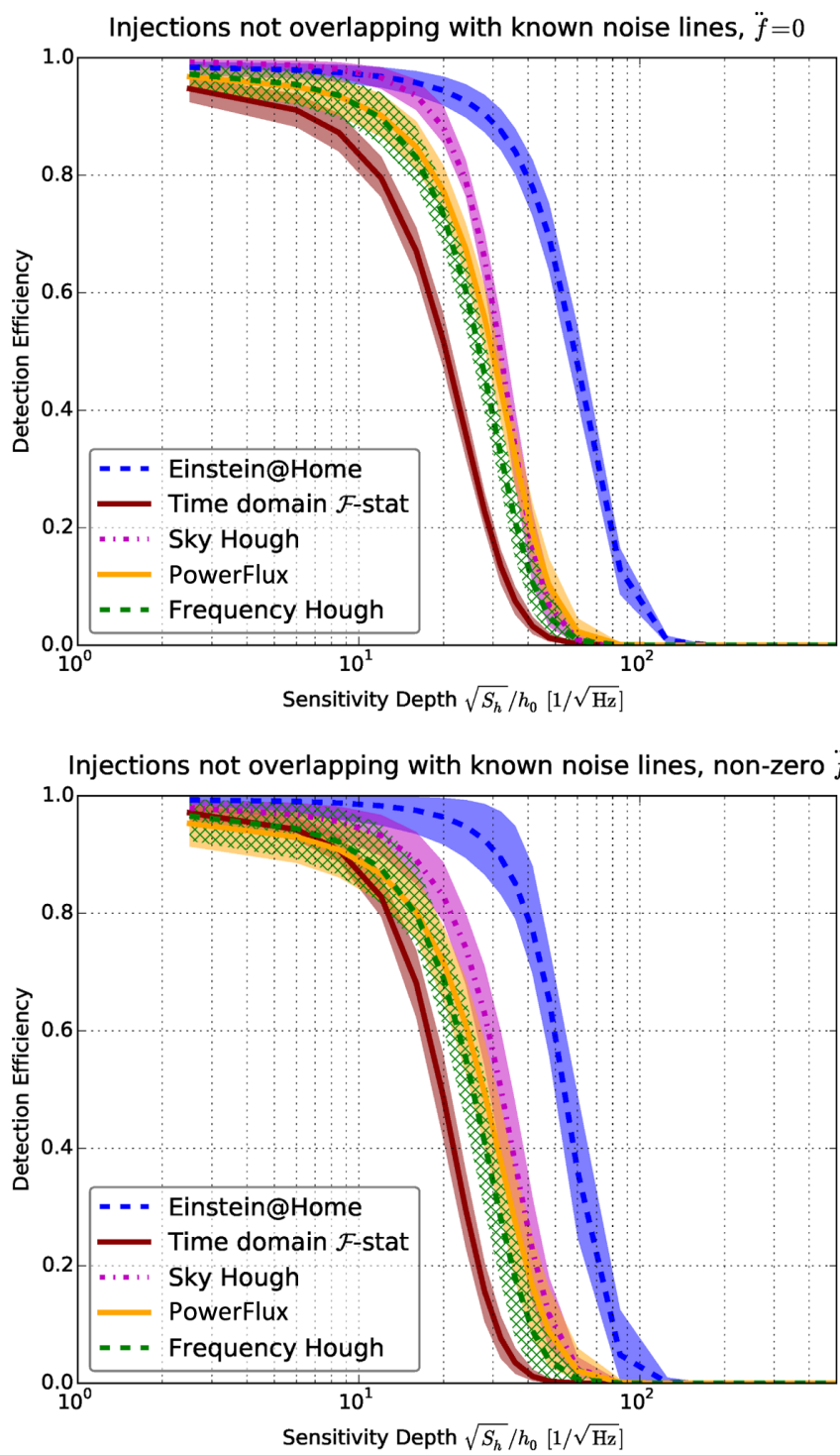

FIG. 7. Detection efficiency measured for injections with zero (2329 injections) and nonzero (781 injections) second order spindown. Frequency Hough results are shown with a hatched error band because the efficiency is measured for a subset of the MDC injections. The curves and error bands are obtained by fitting sigmoids to the data; see Sec. VII A. The error bands represent the statistical uncertainty on the detection efficiency measured for the search implementation and data used in the MDC.

\section{B. Signal parameter recovery}

The distance between the signal and recovered candidates is of interest as it determines the region in parameter space around each candidate that must be searched by the next refinement stage. Also, it serves as a useful cross-check that candidates being claimed as detections in the MDC are within a reasonable distance of the signal parameters.

PowerFlux, Einstein@ Home, and frequency Hough can have more than one candidate per injection. For strong signals, there are in fact many detection candidates around the signal's true parameter values. We study the distribution for the distances of candidates from the true signal parameter values for two sets of candidates: the one with the highest SNR and the one that is closest, in frequency, to the signal.

The distance, in frequency, spin-down, and sky position, is shown for the first set of candidates in Figs. 8 and 9. Here the distance in sky is represented by $\mathrm{dR}$, the angular separation between two sky positions in radians. The $\mathrm{dR}$ scales approximately proportional to the frequency of the signal. There are candidates from PowerFlux and the time domain $\mathcal{F}$-statistic that are not shown because they lie outside the limits of the x-axis. For PowerFlux, these amount to $4 \%, 5 \%$, and $0.6 \%$ of candidates outside the boundaries in frequency, spin-down, and dR, with $<0.1 \%$ of candidates outside all three boundaries. For the time domain $\mathcal{F}$-statistic, $27 \%$ are $>4 \times 10^{-3} \mathrm{~Hz}$ from the signal while $3 \%$ are $>4 \times 10^{-10} \mathrm{~Hz} / \mathrm{s}$. No candidates are outside both boundaries. The PowerFlux method recovers candidates up to $0.02 \mathrm{~Hz}, 1.5 \times 10^{-9} \mathrm{~Hz} / \mathrm{s}$, or $\mathrm{dR}=1.5$ from the injected parameters. The time domain $\mathcal{F}$-statistic recovers candidates up to $0.06 \mathrm{~Hz}, 1.5 \times 10^{-9} \mathrm{~Hz} / \mathrm{s}$, or $\mathrm{dR}=0.4$ from the injection. This means the region these searches need to search to recover the signal from these candidates is larger by a factor of $\mathrm{O}(10)$ in each dimension than the other searches. The time domain $\mathcal{F}$-statistic can afford to do this because they expect $\sim 10$ false alarms.

PowerFlux expects on the order of 10000 false alarms in an all-sky search. While PowerFlux may refine more than one candidate from a signal, only one of the candidates needs to pass through the refinement stages in order to recover the signal. Therefore, the minimum search region needed to recover the signal is better represented by examining the closest signal in frequency.

The Einstein@Home and frequency Hough refinement searches, on candidates from the initial search, cover a predefined region in parameter space around each candidate. In a real search, candidates from a signal within this region will result in a detection after refinement. Therefore, the MDC detection candidates are required to contain the signal within this region (Sec. VI). Figures 8 and 9 support these choices of refinement parameter space, as the majority of candidates are within a smaller region around the signal and there are few outliers at the bounds of the parameter space.

The sky Hough search expects $<15000$ false alarms in an all-sky search over $1500 \mathrm{~Hz}$, because at most one candidate per $0.1 \mathrm{~Hz}$ is selected. The recovered parameters are close to the signal parameters. This allows for quick turnover of results.

Figures 10 and 11 compare the distance between the signal and recovered candidate, now choosing the nearest candidate in frequency for PowerFlux, frequency Hough, and Einstein@Home. The spread of the PowerFlux results has decreased significantly, with the furthest outliers at $7 \times 10^{-3} \mathrm{~Hz}, 1.5 \times 10^{-9} \mathrm{~Hz} / \mathrm{s}$, or dR 0.5 from the signal, 

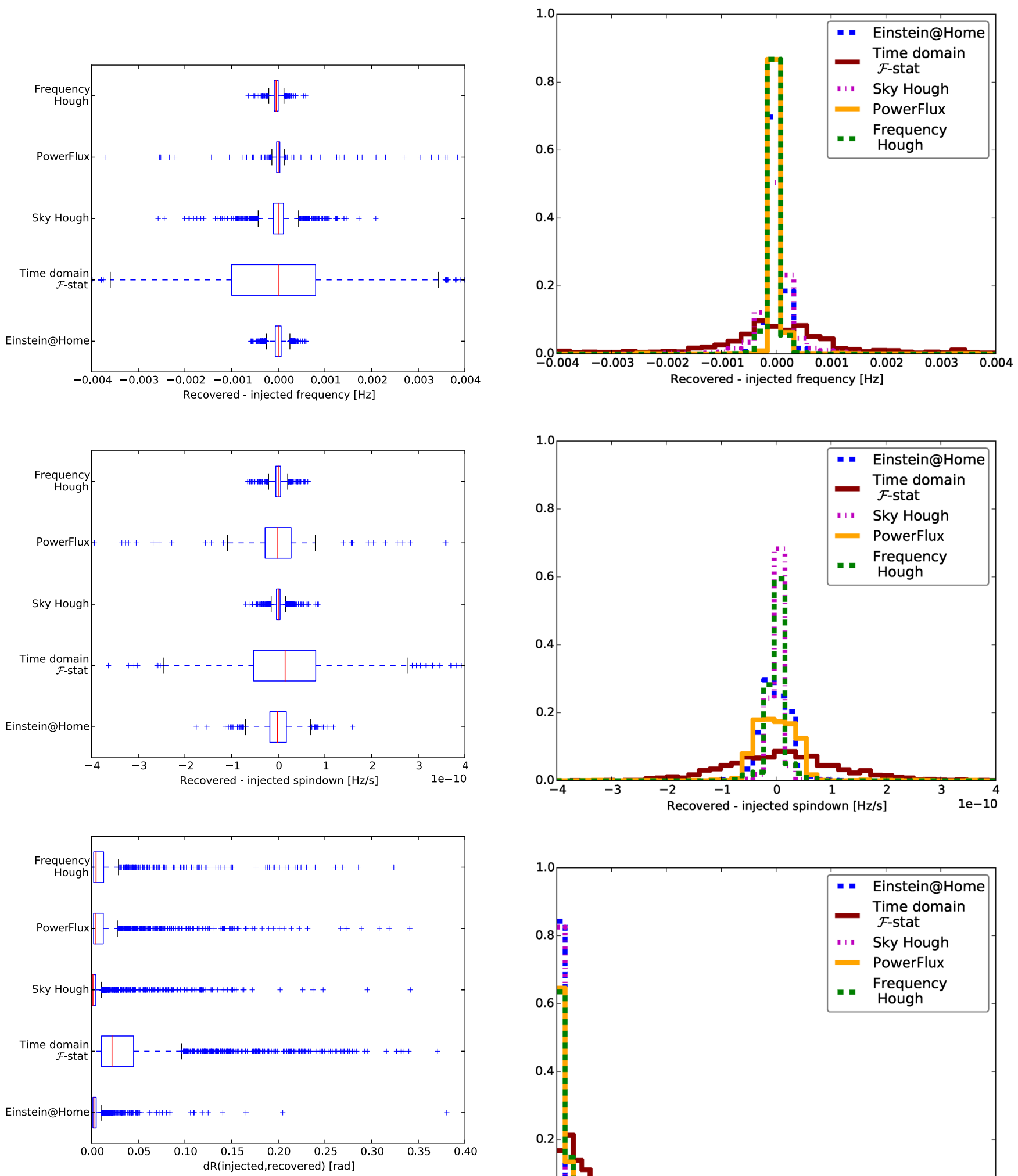

FIG. 8. The distance between the signal and the recovered candidate, in frequency, spin-down, and sky position, when the candidate with the highest SNR is chosen. The red line is the median. The blue box begins and ends at the first and third quartile, respectively. The vertical black bars (whiskers) extend 1.5 times the inner quartile range outside the blue box. The blue crosses are candidates outside this range.

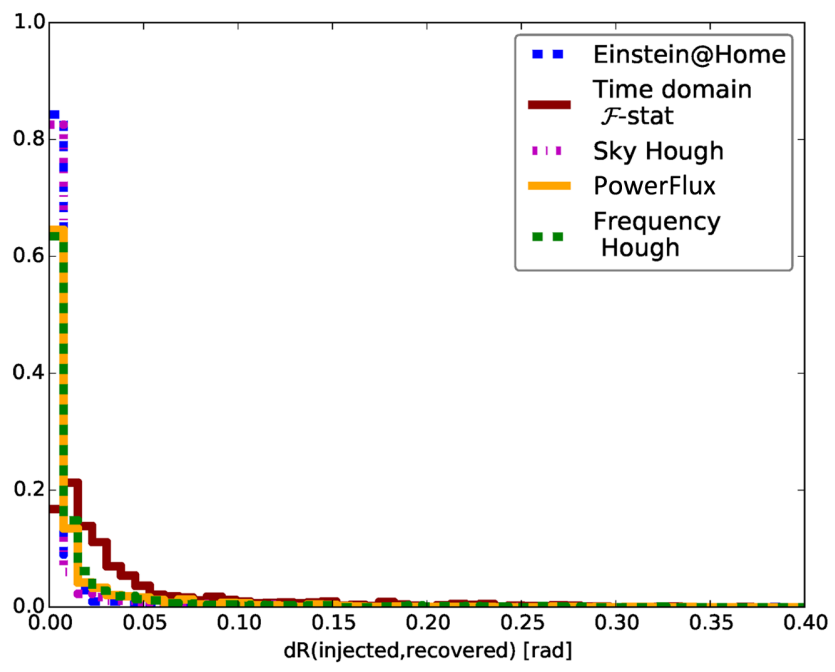

FIG. 9. The distance between the signal and the recovered candidate, in frequency, spin-down, and sky position, when the candidate with the highest SNR is chosen. This is an alternative representation of the same data as in Fig. 8. 

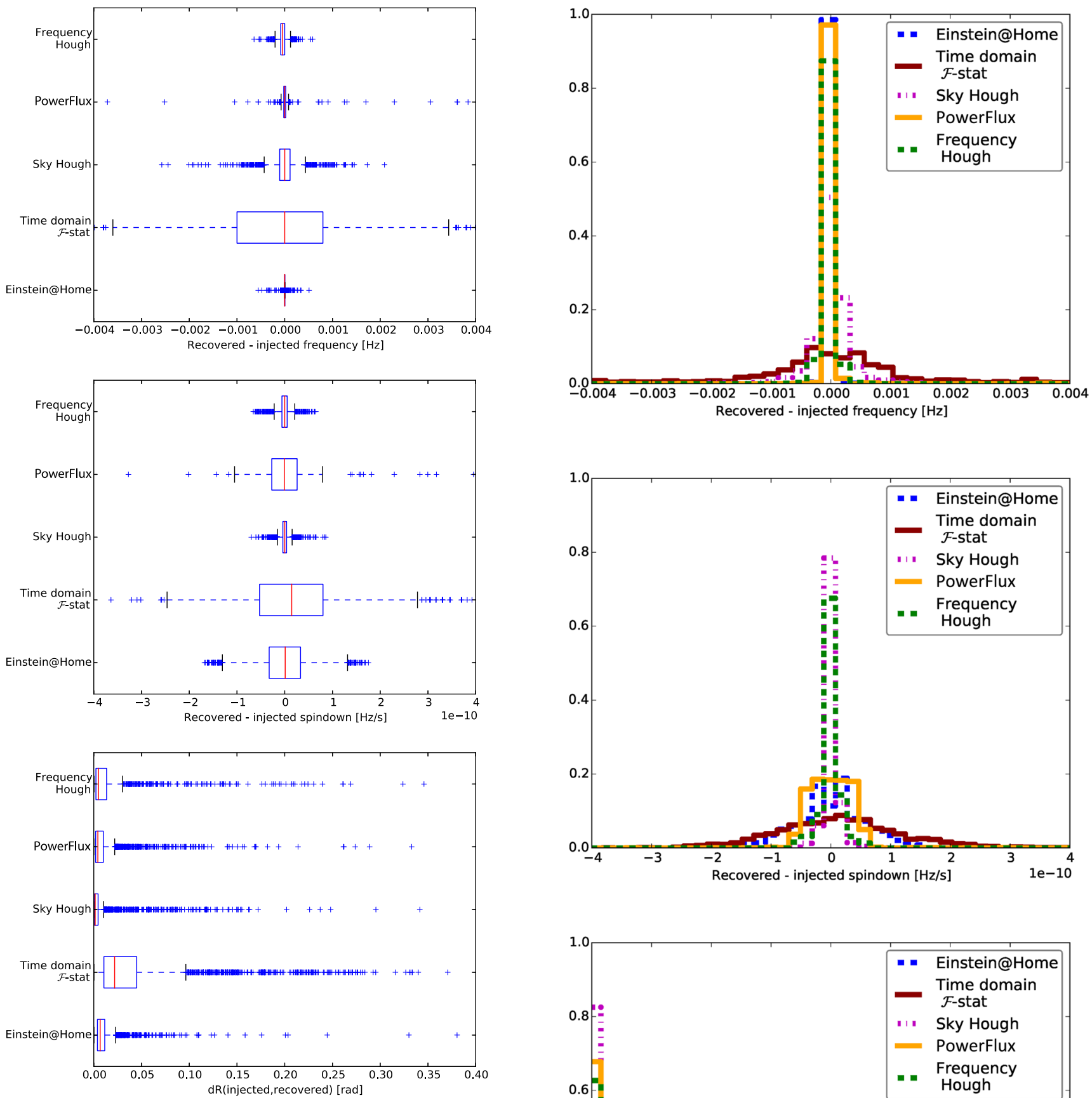

FIG. 10. The distance between the signal and the recovered candidate, in frequency, spin-down, and sky position, when the candidate with the closest frequency to the signal is chosen. The red line is the median. The blue box begins and ends at the first and third quartile, respectively. The vertical black bars (whiskers) extend 1.5 times the inner quartile range outside the blue box. The blue crosses are candidates outside this range.

and less than $1 \%$ of candidates outside the boundaries in any dimension. PowerFlux has demonstrated, in the search of S6 data [15], that they are able to perform refinement searches on all candidates above threshold.

The frequency Hough results are unchanged, as only a handful of signals result in more than one detection
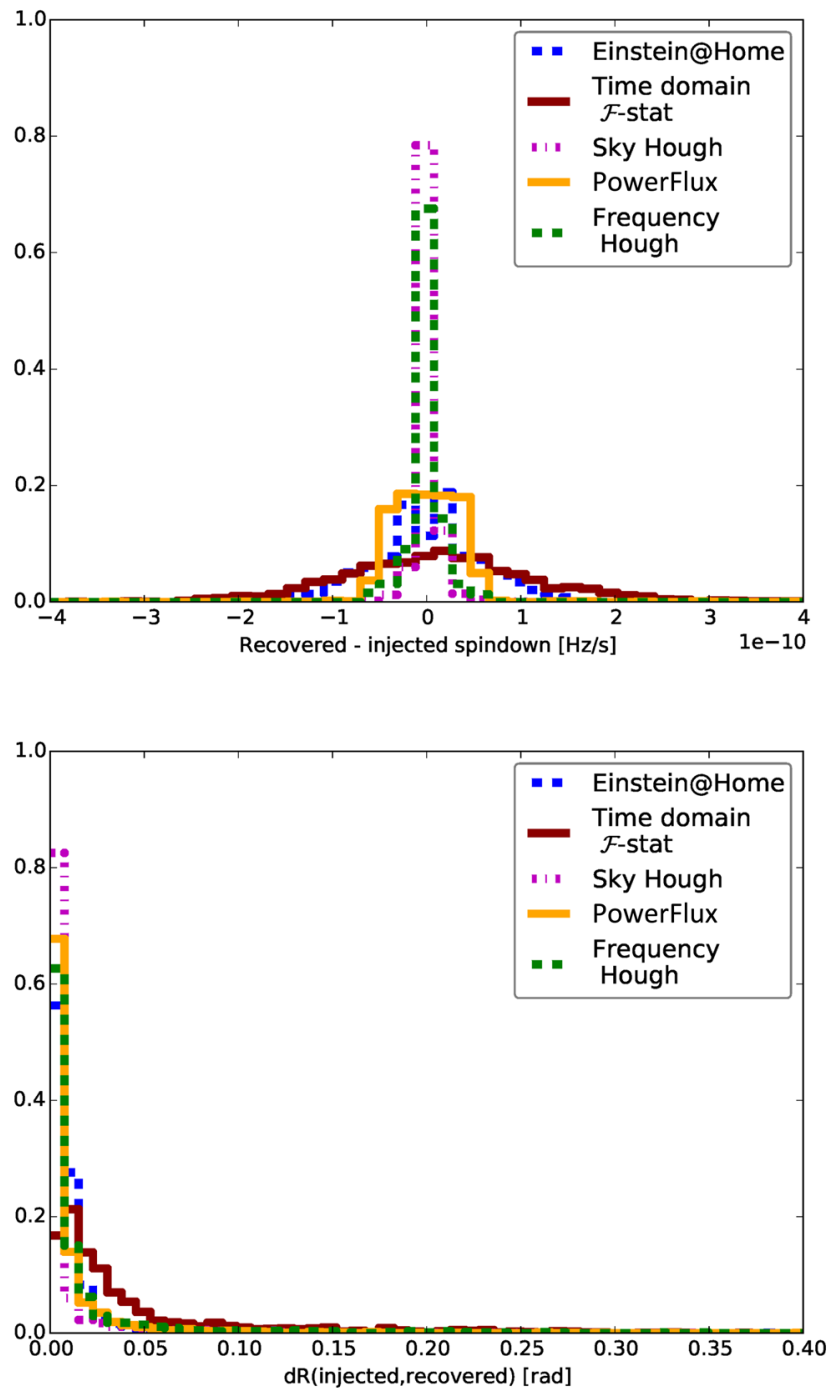

FIG. 11. The distance between the signal and the recovered candidate, in frequency, spin-down, and sky position, when the candidate with the closest frequency to the signal chosen. This is an alternative representation of the same data as in Fig. 10. 
candidate. The inner quartile of the Einstein@Home distribution has changed. However, the presence of candidates at the edge of the refinement region shows that the parameter space cannot be reduced without losing detection efficiency.

\section{CONCLUSION}

We have considered five search pipelines currently performing blind all-sky searches for continuous waves from isolated NSs in advanced detector data. An overview of each pipeline has been presented with regards to the semicoherent method, noise handling, computing cost, and the hierarchical refinement procedure.

To compare the methods, a MDC was performed with 3000 simulated signals. Each pipeline has presented the recovered signal candidates, from which the detection efficiency has been calculated. These results were used to compare the pipelines in quiet and noisy data and to check for dependence on signal frequency and frequency derivatives.

The search methods used by each pipeline make different tradeoffs in sensitivity vs robustness against deviations from the assumed signal model. In this MDC, the detection efficiency is measured for a strictly continuous and phase coherent wave signal. This serves as a first benchmark in the comparison of these five all-sky pipelines. For a comprehensive comparison, the MDC must be extended to include signals that deviate from this signal model, for example, if the NS glitches or if it has a long-period companion [5].

The precision with which the pipelines can be compared is restricted by the dependence of detection efficiency on the observing time of the data and the search configuration, which changes depending on the data available and the parameter space covered. Pipelines are also developing improvements, which will change the detection efficiency of future searches.

With these caveats in mind, we compare the detection efficiency of the pipelines for a standard CW signal. We find similar performance among the sky Hough, PowerFlux, and frequency Hough searches. The detection efficiency for these signals is lower for the time domain $\mathcal{F}$-statistic search. The Einstein @Home search achieved comparable detection efficiency to the other pipelines for signals that are a factor of two weaker, for frequencies below $1000 \mathrm{~Hz}$. The different noise handling approaches left the sky Hough and time domain $\mathcal{F}$-statistic efficiencies unchanged in the presence of known noise lines, while the Einstein@Home and PowerFlux searches lost efficiency. The apparent dependence of detection efficiency on signal frequency for Einstein@Home and PowerFlux is understood. There is no measured dependence on spin-down for any search method.

Despite not explicitly searching over second order spindown, the detection efficiency is unaffected by signals with nonzero second order spin-down. This has been verified for $\ddot{f}<\ddot{f}_{\text {critical }}$. Assuming a standard NS model, $\ddot{f}$ is not expected to exceed $\ddot{f}_{\text {critical }}$ for the sky Hough or frequency Hough searches. The $\ddot{f}$ may exceed $\ddot{f}_{\text {critical }}$ for the other searches. The impact of this has not been examined.

This study is a first step towards a quantitative comparison of the different pipelines. Future studies are needed that include signals deviating from the standard CW model to understand and highlight the benefits of each pipeline.

\section{ACKNOWLEDGMENTS}

The authors would like to thank Erin Macdonald for starting the setup of the MDC, and the LIGO and Virgo CW group for useful insights and discussions. The authors gratefully acknowledge support from the following grants. The Einstein@Home and PowerFlux teams acknowledge the National Science Foundation Grants No. NSF PHY 1104902 and No. NSF PHY 1505932, respectively. The Einstein@Home team also acknowledges the support of the Max-Planck-Society, and the computing resources of the ATLAS super-computing cluster at the Max-PlanckInstitut für Gravitationsphysik/Leibniz Universität Hannover. The time domain $\mathcal{F}$-statistic is supported by the National Science Centre of Poland Grant No. UMO2014/14/M/ST9/00707 and in part by the PL-Grid infrastructure. Sky Hough is supported by the Spanish Ministerio de Economía y Competitividad (Grants No. FPA2013-41042-P, No. CSD2009-00064, No. FPA2015-69815-REDT, No. FPA2015-68783-REDT, and No. FPA2016-76821-P); the Conselleria d'Innovació, Recerca i Turisme del Govern de les Illes Balears, and the European Union FEDER funds. M. P. is funded by the Science and Technology Facilities Council under Grant No. ST/L000946/1. The authors gratefully acknowledge the Italian Istituto Nazionale di Fisica Nucleare. This paper was assigned LIGO document number P1600128. 
[1] LIGO Scientific Collaboration, LIGO: The Laser Interferometer Gravitational-Wave Observatory, Rep. Prog. Phys. 72, 076901 (2009).

[2] LIGO Scientific Collaboration and Virgo Collaboration, Characterization of the LIGO detectors during their sixth science run, Classical Quantum Gravity 32, 115012 (2015).

[3] Virgo Collaboration, Status of Virgo, Classical Quantum Gravity 25, 114045 (2008).

[4] LIGO Scientific Collaboration and Virgo Collaboration, Results of the deepest all-sky survey for continuous gravitational waves on LIGO S6 data running on the Einstein@ Home volunteer distributed computing project, arXiv:1606.09609 [Phys. Rev. D (to be published)].

[5] LIGO Scientific Collaboration and Virgo Collaboration, All-sky search for periodic gravitational waves in the full S5 LIGO data, Phys. Rev. D 85, 022001 (2012).

[6] LIGO Scientific Collaboration and Virgo Collaboration, First low frequency all-sky search for continuous gravitational wave signals, Phys. Rev. D 93, 042007 (2016).

[7] LIGO Scientific Collaboration and Virgo Collaboration, Implementation of an F-statistic all-sky search for continuous gravitational waves in Virgo VSR1 data, Classical Quantum Gravity 31, 165014 (2014).

[8] LIGO Scientific Collaboration and Virgo Collaboration, Application of a Hough search for continuous gravitational waves on data from the fifth LIGO science run, Classical Quantum Gravity 31, 085014 (2014).

[9] C. Messenger et al., Gravitational waves from Scorpius X-1: A comparison of search methods and prospects for detection with advanced detectors, Phys. Rev. D 92, 023006 (2015).

[10] P. Jaranowski, A. Krolak, and B. F. Schutz, Data analysis of gravitational-wave signals from spinning neutron stars: The signal and its detection, Phys. Rev. D 58, 063001 (1998).

[11] B. Owen, How to adapt broad-band gravitational-wave searches for $r$-modes, Phys. Rev. D 82, 104002 (2010).

[12] S. Frasca, P. Astone, and C. Palomba, Evaluation of sensitivity and computing power for the Virgo hierarchical search for periodic sources, Classical Quantum Gravity 22, S1013 (2005).

[13] P. R. Brady and T. Creighton, Searching for periodic sources with LIGO. II. Hierarchical searches, Phys. Rev. D 61, 082001 (2000).

[14] LIGO Scientific Collaboration, All-sky search for periodic gravitational waves in LIGO S4 data, Phys. Rev. D 77, 022001 (2008).

[15] B. P. Abbott et al. (LIGO Scientific Collaboration and Virgo Collaboration), Comprehensive all-sky search for periodic gravitational waves in the sixth science run LIGO data, Phys. Rev. D 94, 042002 (2016).
[16] V. Dergachev, On blind searches for noise dominated signals: A loosely coherent approach, Classical Quantum Gravity 27, 205017 (2010).

[17] P. Astone, K. M. Borkowski, P. Jaranowski, M. Piętka, and A. Królak, Data analysis of gravitational-wave signals from spinning neutron stars. V. A narrow-band all-sky search, Phys. Rev. D 82, 022005 (2010).

[18] http://einsteinathome.org.

[19] H. J. Pletsch and B. Allen, Exploiting Large-Scale Correlations to Detect Continuous Gravitational Waves, Phys. Rev. Lett. 103, 181102 (2009).

[20] D. Keitel, R. Prix, M. A. Papa, P. Leaci, and M. Siddiqi, Search for continuous gravitational waves: Improving robustness versus instrumental artifacts, Phys. Rev. D 89, 064023 (2014).

[21] M. A. Papa et al., Hierarchical follow-up of sub-threshold candidates of an all-sky Einstein@Home search for continuous gravitational waves on LIGO sixth science run data, arXiv:1608.08928 [Phys. Rev. D (to be published)].

[22] F. Antonucci, P. Astone, S. D'Antonio, S. Frasca, and C. Palomba, Detection of periodic gravitational wave sources by Hough transform in the $f$ versus $\dot{f}$ plane, Classical Quantum Gravity 25, 184015 (2008).

[23] P. Astone, A. Colla, S. D'Antonio, S. Frasca, and C. Palomba, Method for all-sky searches of continuous gravitational wave signals using the frequency-Hough transform, Phys. Rev. D 90, 042002 (2014).

[24] LIGO Scientific Collaboration and Virgo Collaboration, Characterization of the LIGO detectors during their sixth science run, Classical Quantum Gravity 32, 115012 (2015).

[25] LSC Algorithm Library Suite, https://www.lsc-group.phys .uwm.edu/daswg/projects/lalsuite.html.

[26] K. Wette et al., Searching for gravitational waves from Cassiopeia A with LIGO, Classical Quantum Gravity 25, 235011 (2008).

[27] C. Palomba, Pulsars ellipticity revised, Astron. Astrophys. 354, 163 (2000).

[28] M. Shaltev, P. Leaci, M. A. Papa, and R. Prix, Fully coherent follow-up of continuous gravitational-wave candidates: An application to Einstein@Home results, Phys. Rev. D 89, 124030 (2014).

[29] R. Prix and M. Shaltev, Search for continuous gravitational waves: Optimal StackSlide method at fixed computing cost, Phys. Rev. D 85, 084010 (2012).

[30] M. Oliver et al., Improvement and characterization of the Hough search for all-sky continuous gravitational wave signals (to be published). 